\title{
Editorial
}

\section{Weather and Climate in Monsoon Regions}

\author{
Lin Wang, ${ }^{1}$ June-Yi Lee, ${ }^{2}$ Haishan Chen, ${ }^{3}$ Fred Kucharski, ${ }^{4}$ Xiaolong Jia, ${ }^{5}$ \\ Xiaojing Jia, ${ }^{6}$ and Jieshun $\mathrm{Zhu}^{7}$ \\ ${ }^{1}$ Center for Monsoon System Research, Institute of Atmospheric Physics, Chinese Academy of Sciences and \\ Joint Center for Global Change Studies, Beijing 100190, China \\ ${ }^{2}$ Institute of Environmental Studies, Pusan National University, Busan, Republic of Korea \\ ${ }^{3}$ School of Atmospheric Sciences, Nanjing University of Information Science and Technology, Nanjing 210044, China \\ ${ }^{4}$ Earth System Physics Section, Abdus Salam ICTP, Trieste, Italy \\ ${ }^{5}$ National Climate Center, China Meteorological Administration, Beijing 100081, China \\ ${ }^{6}$ Department of Earth Sciences, Zhejiang University, Hangzhou 310027, China \\ ${ }^{7}$ Climate Prediction Center, NOAA/NWS/NCEP, College Park, MD, USA
}

Correspondence should be addressed to Lin Wang; wanglin@mail.iap.ac.cn

Received 27 July 2015; Accepted 27 July 2015

Copyright (C) 2015 Lin Wang et al. This is an open access article distributed under the Creative Commons Attribution License, which permits unrestricted use, distribution, and reproduction in any medium, provided the original work is properly cited.

Monsoon regions feed about 70 percent of the world's population. The weather and climate variability in these regions often lead to natural hazards such as flooding, droughts, heat waves, and blizzards, which exert strong impacts on both the society and human lives. This is particularly the case over many Asian monsoon regions, where the countries are less developed and the people heavily depend on agriculture. As a result, the monsoon research has traditionally held a very high priority for the international scientific communities of weather and climate. It is an urgent task for the community to gain a deeper understanding of the monsoon variability to meet the rising demand of a better weather forecast and climate prediction.

This special issue consists of seven research papers, all of which focus on the Asian monsoon. Two papers are case studies on the 2013 summer monsoon in China, where both extreme rainfall and heat waves were recorded in the middle and lower reaches of the Yangtze River Valley. One paper discusses the stratospheric and extratropical influences on the East Asian monsoon. The rest of papers discuss the spatiotemporal variability, mechanism, and prediction of the Asian monsoon over China, India, and Malaysia through statistical and diagnostic approaches. Besides the monsoon rainfall, the evaporation that is crucial to understand the hydrological cycles in monsoon regions and the cold spell that is another important aspect of the monsoon climate are also discussed.

All seven papers in this special issue can be categorized as traditional climate studies. In recent years, an increasing number of scientists from the traditional weather and climate communities are working together and trying to fill the gap of understanding and prediction between the weather and climate. A Subseasonal to Seasonal (S2S) Prediction Project was launched in 2013 under the umbrella of the World Weather Research Program (WWRP), the World Climate Research Program (WCRP), and the Observing System Research and Predictability Experiment (THORPEX), aiming to promote a seamless weather/climate prediction. We sincerely expect that a better and more integrated understanding of weather and climate of monsoon regions could be obtained in the near future.

Lin Wang June-Yi Lee Haishan Chen Fred Kucharski Xiaolong Jia Xiaojing Jia Jieshun Zhu 

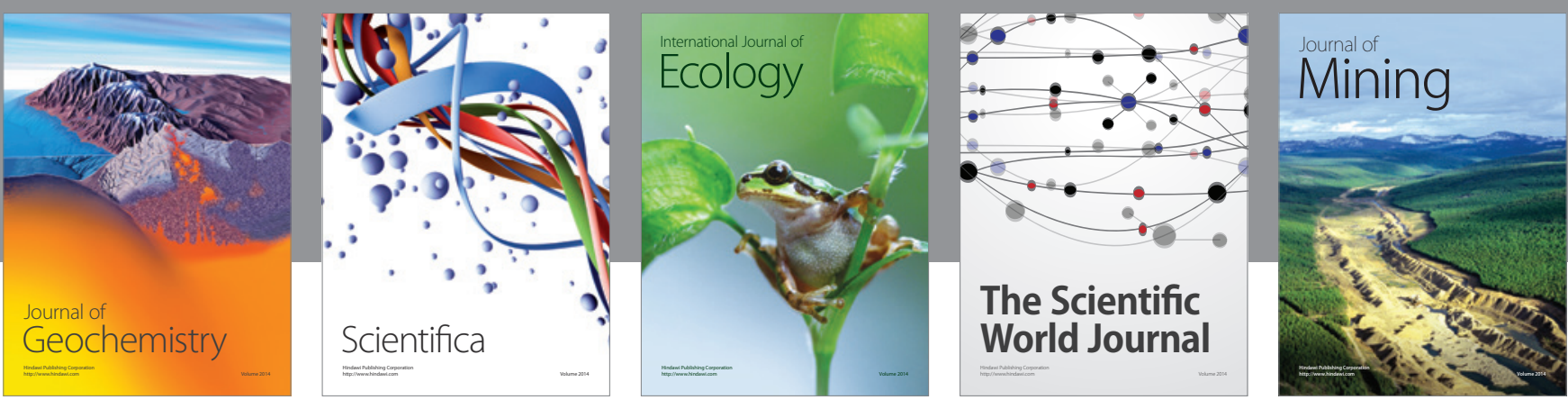

The Scientific World Journal
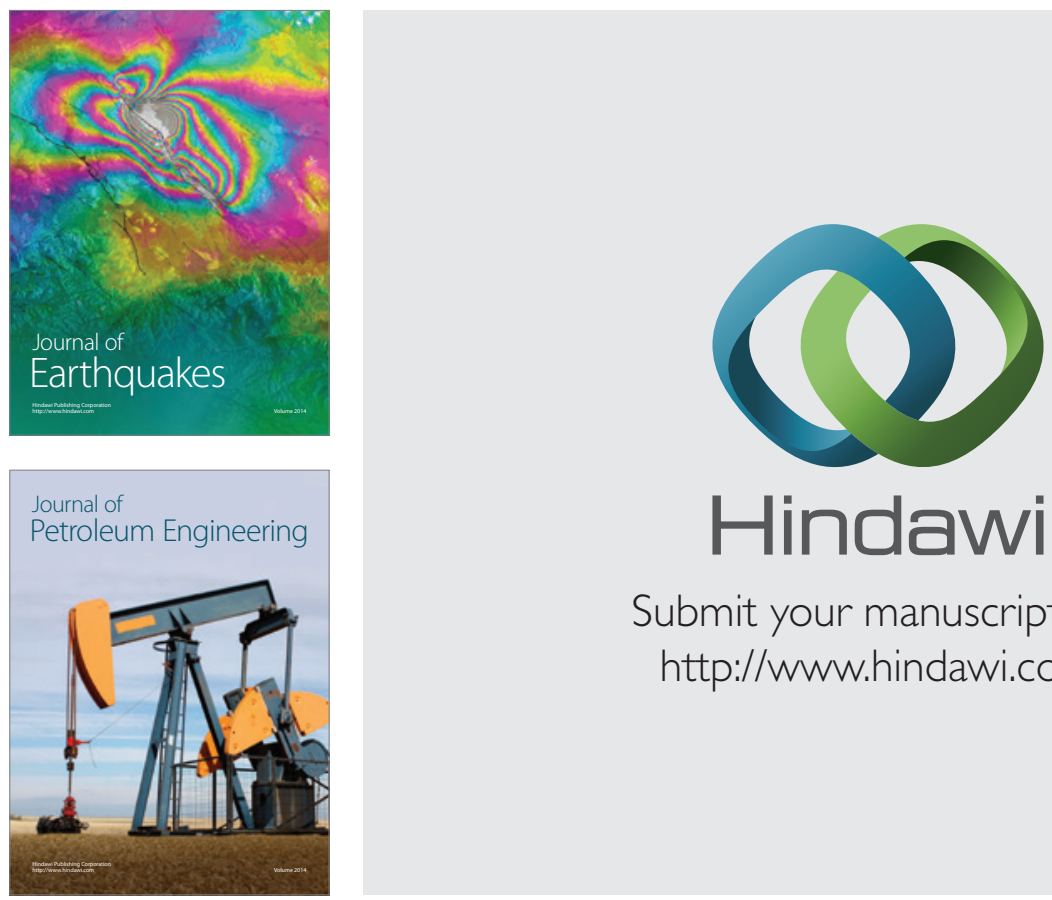

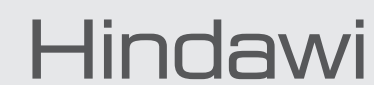

Submit your manuscripts at

http://www.hindawi.com
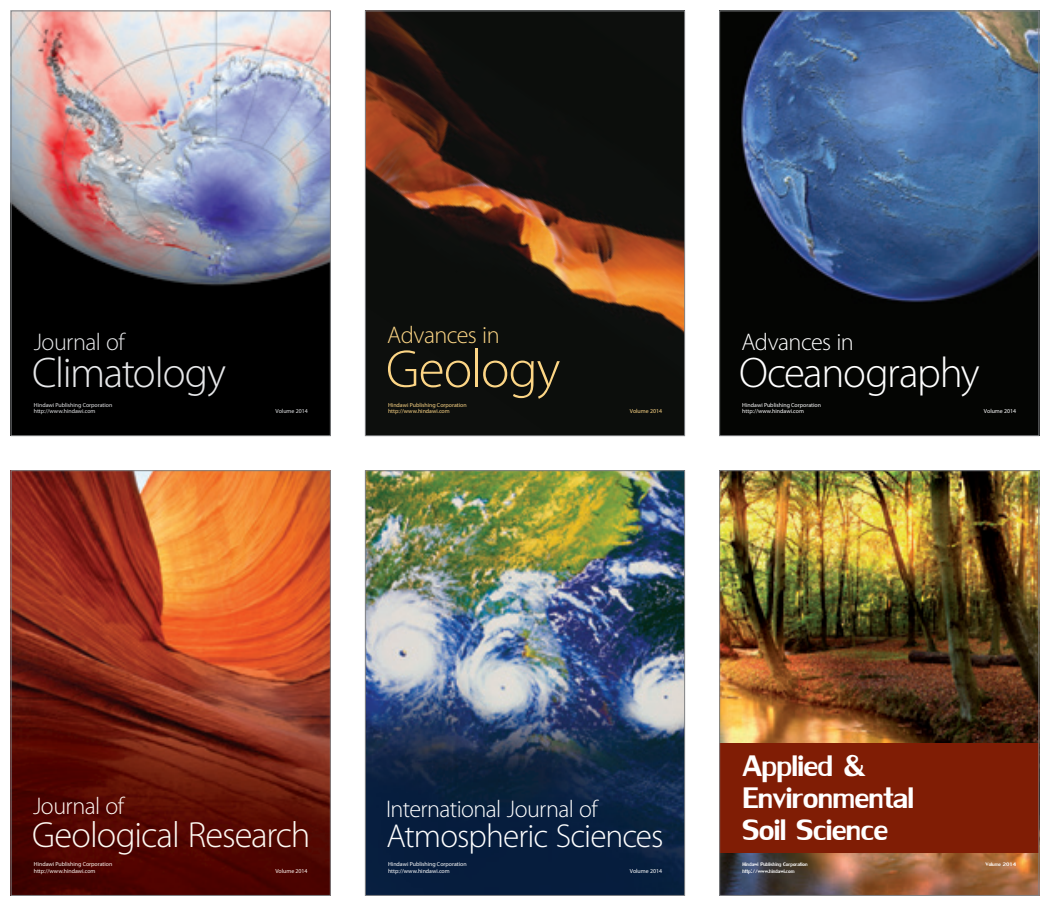
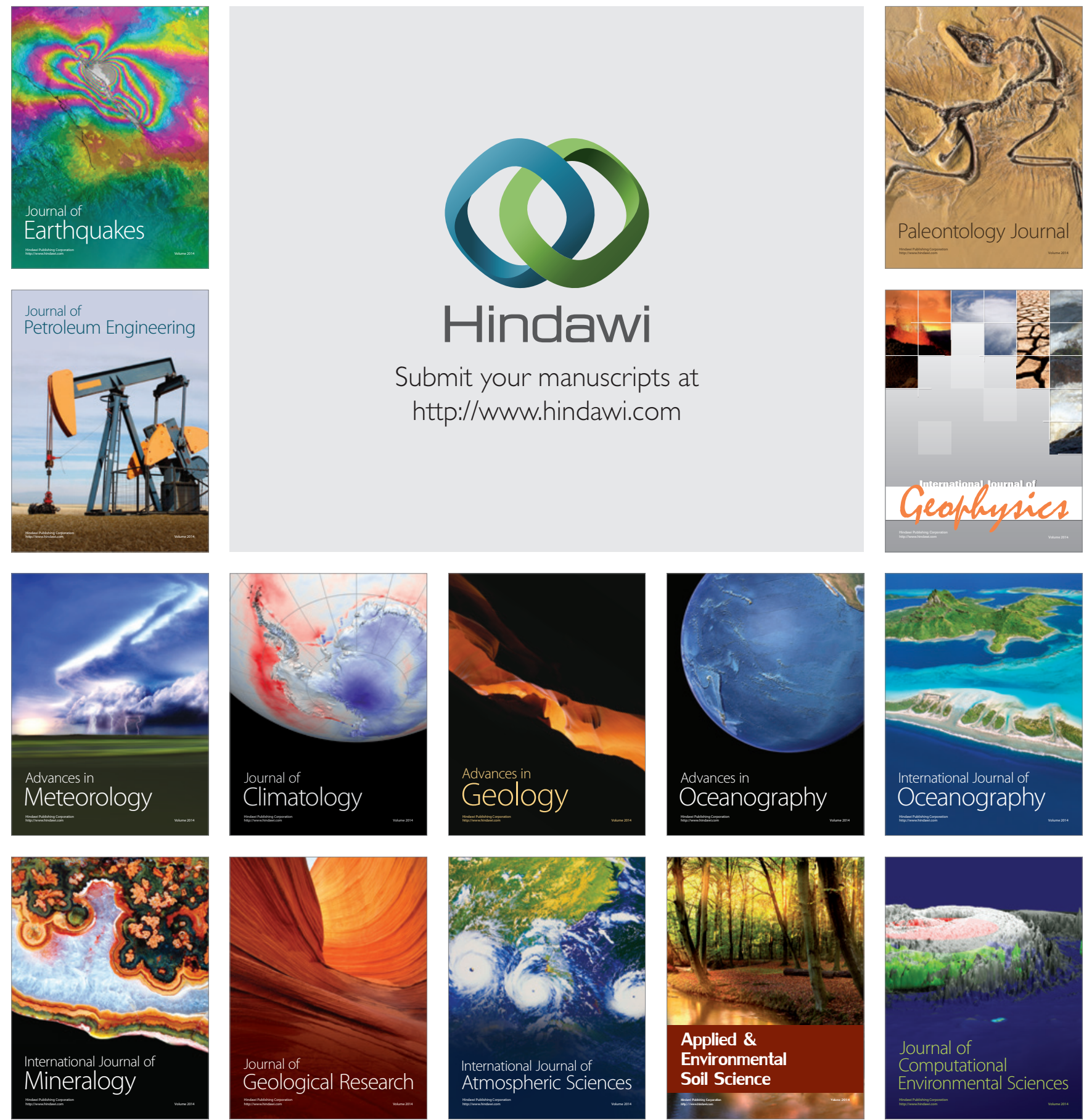